\title{
BREAST CANCER DETECTION USING MARK RCNN SEGMENTATION AND ENSEMBLE CLASSIFICATION WITH FEATURE EXTRACTION
}

\author{
Prasath Alias Surendhar $\mathrm{S}^{1^{*}}$, R.Vasuki ${ }^{2}$ \\ ${ }^{1 *}$ Research Scholar, Dept. of Biomedical Engineering, Bharath Institute of Higher Education and Research, \\ Chennai. \\ ${ }^{2}$ Professor \& Head, Dept. of Biomedical Engineering, Bharath Institute of Higher Education and Research, \\ Chennai. \\ 1*Email: mail.surendhar@gmail.com, ${ }^{2}$ Email: rkaran02@gmail.com
}

\begin{abstract}
Breast cancer continues to be the common cancer which causes more decease among women and about more than two million cases is identified every year and according to the record 523,000 deaths are caused per year due to breast cancer. Mammographic mass identification and segmentation are accomplished usually as sequential and distinct tasks, where in previous studies segmentation was often manually performed only on true positive cases. Machine learning (ML) approaches have been grown from manually provided inputs to systematic initializations. The developments in ML techniques have produced independent and more intelligent computeraided diagnosis (CAD) systems. Moreover, due to the learning ability, ML techniques have been upgraded constantly. Recently, ML techniques are progressive with deeper and varied representation methods, generally termed as deep learning (DL) techniques, and have produced significant impacts on increasing the ability of diagnosing using CAD systems. So this paper proposes the novel architecture in detecting the breast cancer. Here the input database is mammogram image dataset. Initially this image has been resized by pre-processing process, then this pre-processed image has been segmented using Mask RCNN (Region-based convolution neural networks). Then this segmented image has been sent for extracting the feature using inception V3 and ResNet 152 networks. The ensemble classifier of decision tree and random forest has been used for classification and this classified output is detected based on the color variation and tumor size gives the enhanced accuracy in detecting the cancer.
\end{abstract}

Keywords: Breast cancer, Mammographic mass, Machine learning (ML), pre-processing, Mask RCNN, inception V3, ResNet 152, decision tree, random forest.

\section{INTRODUCTION}

One of the most commonly diagnosed cancers by women worldwide is breast cancer. Mammography is considered as the basic method for breast screening, where fatigue and missing detections in human examination can arise from the vast amount of data involved. CAD systems were designed as a 'second pair of eyes' in interpreting mammography in order to support radiologists and improve the detection confidence [1]. The CAD system, at first, typically extracts candidate regions from breast mass and classifies these regions as either normal or abnormal depending on the extracted features. Conventional CAD systems focus primarily on generating unsupervised candidate regions along with hand-crafted features for detecting breast masses [2].

In the recent days, biomedical along with nanotechnology have been used to provide the greatest possible degree of advancement towards human health care.Nano-material shall therefore become one of the goods with the size of 1 to $1000 \mathrm{~nm}(10-9 \mathrm{~m})$ [3]. As stated by World Health Organisation (WHO), the most prevalent cancer found in women worldwide is breast cancer. One affected women for every three cases lose their life. Current investigation techniques for breast cancer include various methods like magnetic resonance imaging (MRI), mammography and pathological tests. In these approaches, the standard histopathological images enhance the diagnostic accuracy with patients previously investigated with mammograms. In addition, this assessment provides more accurate and reliable information on the diagnosis of cancer and the evaluation of its effects on the tissues around it [4].

In medical diagnosis, machine learning techniques and expert systems are extensively used. In fact, the most significant factors for diagnosis is the assessment of patient data and the decisions made by the experts. Various 
Artificial Intelligence and expert systems can assist diagnosis. Automated diagnostic systems help the medical experts made to avoid the errors and diagnosis data more quickly and deeply. Training sets can also include both abnormal and normal images obtained from the mammography scan, Ultrasound scans, or else in biopsy data. The machine can process only grey scale image and to extract image without noise or artifact we are using different filters to remove the artifact [5].

At this point, numerous procedures exist for detecting cancer in humans such as X-ray mammogram, ultrasound, MRI, biopsy and Positron Emission Tomography, etc. Even though several techniques exist, only experience physicians are involved in diagnosis. When compared to this, involving machine learning approaches an improve the accuracy of detection. Classifiers can help the physicians to diagnose more precisely by minimizing errors. The J48 decision tree is the most common classifier involved whose advantages are

- Can be constructed easily

- More flexible

- Debugging is easier

- Can be applied for numerical and categorical data

- $\quad$ Suitable for regression and classification models

Boosting/Bagging methods exists to ensemble different classifiers for classifying efficiently. Several exiting classification methods are expensive and complex. In this proposed method, priority is set for various attributes in the dataset. Therefore, the priority of the attributes is also considered along with the information gain during classification.

Classification is one of the most extensively used decision-making task in machine-based learning algorithms. The major aim of classification is to accurately predict the target class for every data sample. In training phase of classification, every sample data has predefined target class. Whereas while testing, unknown test instances are predicted using the model constructed for training set. Classifying algorithms involves huge volume of data and the classification is based on the training set[7].

The remaining part of the paper is presented as follows. Section 2 reviews the background works. Section 3 elaborates the proposed model. Section 4 discusses the obtained experimental result after diagnosing and predicting breast cancer. Section 5 concludes this research work and outlines the future enhancements to be carried out.

\section{RELATED WORKS}

Recent advancements of deep learning (DL) techniques solve the issues of detection an classification as these techniques are more robust. These techniques involve convolutional neural networks (CNNs) to directly observe useful features from the training set and remarkable results were observed. Dhungel et al. [8] developed a multiscale deep belief network and Gaussian mixture model for detecting candidate region which were then classified by the hybrid approach which integrated R-CNN and random forests. Li et al. [9] used an unsupervised region detecting approach which involved morphological analysis and the candidate regions were classified using CNN. But, these CAD systems performs only detection and do not involve segmentation. A separate approach is applied for segmentation. Dhungel et al. [10] extended their work in [8] by introducing a segmentation method based on conditional random field and a level set scheme. This model performed only segmentation on true positive (TP) detections while the false positive (FP) detections has to be manually rejected. Oliveira et al. [11] adopted residual neural network (ResNet) for detecting candidate regions and CNN approach for classifying them. Segmentation refinement strategy was used for generating contour of masses. The authors of [10] and [11] considered mass detection and segmentation as distinct tasks and compriseof multiple deep learning networks which has to be sequentially fine-tuned. Still, there is a demand for more integrated model which can efficiently perform mass detection and segmentation simultaneously.

Songet al [12] analysed the effectiveness of different decision tree algorithms like CHAID, C4.5, CART, and QUEST by the use of SAS and SPSS. AlSahilyet al[13] studiedand analysed the performance of various decision tree algorithms such as Function Tree, J48,Alternating Decision (AD) Tree, Random Forest Tree, Decision stump and Best First. It was observed that Function Tree classifier produced the precision of $97.7 \%$ with andDecision stump with a precision of $88 \%$. The former correctly identified more instances while the identified only less instances. Yadavet al [14] performed a comparative analysis of decision tree and Artificial Neural Network (ANN) which involved breast cancer data It was observed that the overall prediction accuracy produced by decision tree was from $90 \%$ to $94 \%$, and for ANN was from $94.5 \%$ to $97 \%$. Bhargavaet al [15] investigated the efficiency of Decision Trees against Univariate and Multivariate methods. It was proved that Multivariate Decision Tree produced better results than Univariate approach. Kauret al [16]used WEKA, a data mining tool, as an API of MATLAB to construct the modified version of J48 classifier. Extened version produced an accuracy of $99.87 \%$ than the existing $\mathrm{J}-48$ classifier. 
Pritom et al [17]investigated few classifiers like Naive Bayes (NB), C4.5 Decision Tree and SVM (Support Vector Machine) for predicting the recurrence of breast cancer. The features were ranked and the attributes were reduced based on the ranking to increase the prediction accuracy. It was observed that SVM produced outstanding results than other classifiers. The prediction accuracy was based on the selection of feature. Nalini et al [18] analysed the performance of NB and J48 classifier in diagnosing breast cancer. Consistency Subset Evaluator was used for feature selection. NB classifier was proved to bea better classifier. Khadhim[19] employed ID3 tree classifier algorithm for classifying the risk factors of breast cancer and determined the more effective factor. Moreover, the issues in designing a breast cancer detecting system were focused. Chaet al [20] employed Genetic algorithms for selecting best attributes and to develop precisenear-optimum decision trees which were transformed into chromosomes by encoding and decoding a decision tree.

Alickovicet al[21] used Genetic Algorithm on different classifiers which include Decision Trees, Logistic Regression, Bayesian Network, Random Forest, MLP, SVM, RBFN and Rotation Forest for predicting breast cancerbased on feature selection. It was found that Rotation Forest with Genetic Algorithm based on 14 features produced an accuracy of $99.48 \%$. Wang et al [22] coined a novel decision tree based Self adaptive NBTree classifier which integrated Naive Bayes and Decision trees.NBresolves overgeneralization and overspecialization. Fruend et al[23] constructed ADTree which was similar to C5.0with boosting and when its efficiency was analysed, it was found that error rate was identical for both methods. Venketasan et al [24] made a comparative study on decision tree classifiers namely CART, J48, BFTree and ADTree. J48 obtained a high accuracy of 99\%, BFtree with $98 \%$, ADTree with $97 \%$ and CART with $96 \%$.Snousyet al [25] used single and ensemble methods and involved chi-square and gain ratio attribute methods for selecting features. Ensemble methods improved the rate of accuracy. For bagging, approaches like C4.5 and REP Trees and for boosting, approaches like AdaBoost, Random Forests and ADTree were involved.

Prasath Alias Surendhar S distinguishes and arranges MR pictures of cerebrum just into typical and irregular. In view of the trial results, $\mathrm{CNN}$ is considered to real preferences over customary neural systems, because of the way that $\mathrm{CNN}$ gains from the preparation information promptly. This speed of learning gives CNN the capacity of adjusting its learning progressively $[26,27,28]$.

\section{Materials and Methods}

In this section focuses on the materials and methods of this research work. The INbreast dataset is used for this research work which is borrowed from http://medicalresearch.inescporto.pt/breastresearch/index.php/Get_INbreast_Database. At present, this dataset is the first freely available large scale full-digital mammographic dataset with correctly annotated mammograms.115 cases of 410 mammograms are included. In total, 116 breast masses occur within the size range of [15 $\mathrm{mmmm} 2,3689 \mathrm{mmmm} 2]$. The mammograms have a pixel scale of 70 with bit depth as 14-bits. The similar validation data partition is used for assessing INbreast to make a clear comparison, where the dataset is partitioned randomly as $60 \%$ for training, $20 \%$ for testing and $20 \%$ for validation.

These below methods are implemented in this research work.
- VGG-16
- Alex Net
- GoogleNet
- CNN
- SegNet
- R2U-Net
- Proposed_DeepIncepRC

The algorithms are executed using Python 3 with 384 GB DDR4 and an NVIDIA Tesla V100 16GB on a Dell EMC PowerEdge R740 server.

\section{Results and Discussions}

In this section focuses on the results of this research work. The below table gives the parametric comparison of proposed M-RCNN with ensemble classifier. Mask R-CNN performs features extraction and classifies the affected regions. It simultaneously executes identification and segmentation activities and it is possible to optimize the entire network. In this suggested CAD, the mask R-CNN with ensemble classifier does not require any massive training. Just about 10 minutes of 10 epochs is required for every training round. For testing this Mask R-CNN, just only less than $1 \mathrm{~s}$ per image is required. 
Table-1: Parametric comparison of various network models

\begin{tabular}{|c|c|c|c|c|c|}
\hline S.NO & Techniques & Accuracy'(\%) & Precision(\%) & Recall(\%) & F1-score(\%) \\
\hline 1 & VGG-16 & 86.2 & 83.1 & 91.6 & 75.3 \\
\hline 2 & Alex Net & 93.8 & 86.1 & 91.2 & 81.3 \\
\hline 3 & GoogleNet & 95.8 & 89.1 & 89.4 & 89.1 \\
\hline 4 & CNN & 97.1 & 89.1 & 94.3 & 88.8 \\
\hline 5 & SegNet & 96.6 & 86.6 & 81.4 & 84.6 \\
\hline 6 & R2U-Net & 95.5 & 89.3 & 77.9 & 81.7 \\
\hline 7 & Proposed_DeepIncepRC & 97.3 & 97.37 & 97.15 & 97.25 \\
\hline
\end{tabular}

The below diagram represents that the accuracy levels of various classifiers. Such as VGG-16 technique gives $86.2 \%$ of accuracy level. Alex Net technique gives $93.8 \%$ of accuracy level. GoogleNet technique gives $95.8 \%$ of accuracy level. CNN classifier gives the accuracy value is $97.1 \%$, SegNet algorithm gives $96.6 \%$ of accuracy level. R2U-Net classifier gives 95.5\% and Proposed_DeepIncepRC gives 97.3\% of accuracy level.

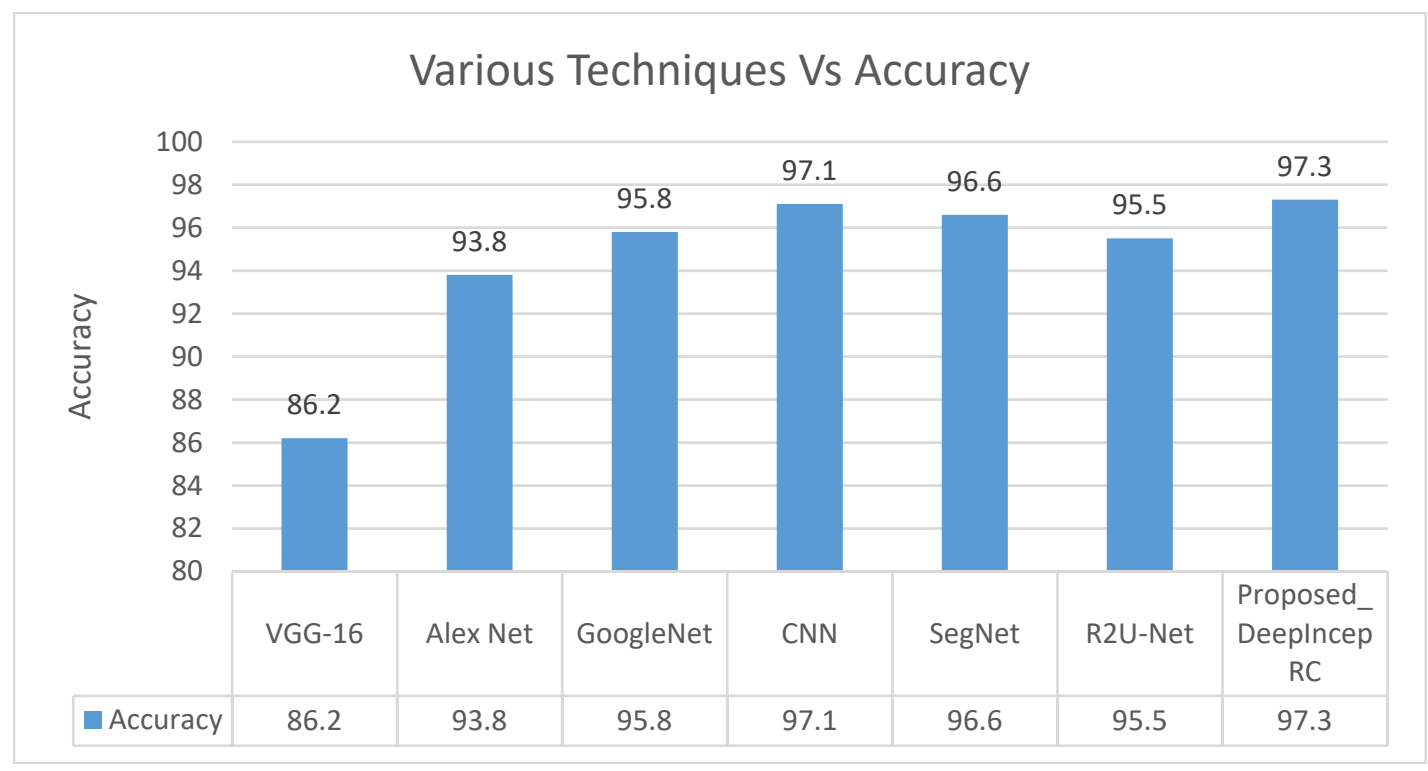

Figure 1: Various Classifiers Vs Accuracy

The above diagram shows that the graphical representation of the various classifiers with their accuracies. GoogleNet,CNN, SegNet,R2U-Net,Proposed_DeepIncepRC are producing above 95\% of accuracy level except VGG-16 classifier which is $86.2 \%$ of accuracy level. The CNN and Proposed_DeepIncepRC have above $97 \%$ of accuracy level.

The below diagram represents that the precision values of various classifiers. like VGG-16 technique gives $83.1 \%$ of precision value. Alex Net technique gives $86.1 \%$ of precision value. GoogleNet technique gives $89.1 \%$ of precision value. CNN classifier gives the precision value is $89.1 \%$, SegNet algorithm gives $86.6 \%$ of precision value. R2U-Net classifier gives the precision value is $89.3 \%$ and Proposed_DeepIncepRC gives $97.37 \%$ of precision value. 


\section{Various Techniques Vs Precision}

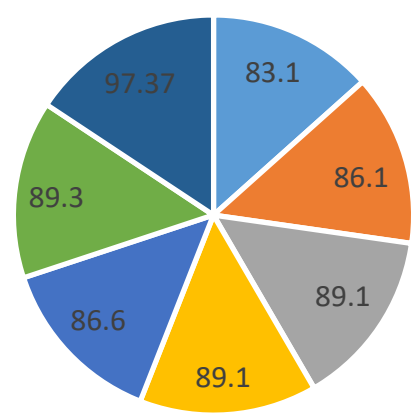

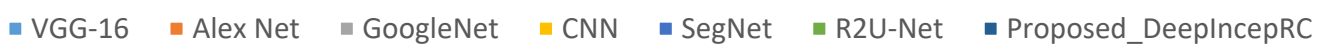

Figure 2: Various Classifiers Vs Precision

The above diagram shows that the precision values of the various classifiers. All the precision values are lies in between $83 \%$ to $90 \%$ but our proposed method Proposed_DeepIncepRC has highest precision value which is $97.37 \%$

The below diagram represents that the recall values of various classifiers. i.e., VGG-16 technique gives $91.6 \%$ of recall value. Alex Net technique gives $91.2 \%$ of recal value. GoogleNet technique gives $89.4 \%$ of recall value. CNN classifier gives the recall value is $94.3 \%$, SegNet algorithm gives $81.4 \%$ of recall value. R2U-Net classifier gives the recall value is $77.9 \%$ and Proposed_DeepIncepRC gives $97.15 \%$ of recall value.

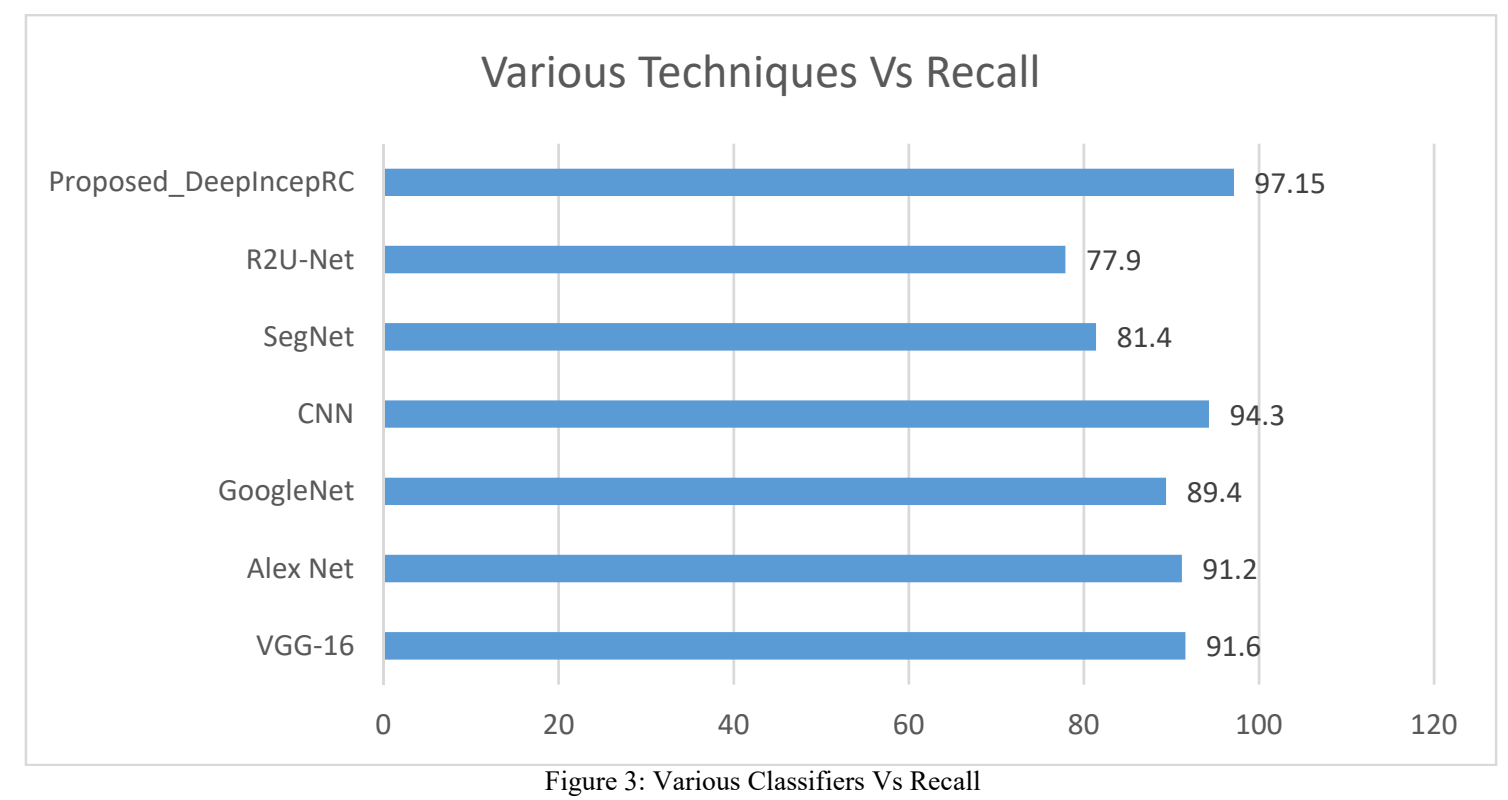

The above diagram shows that the graphical representation of the various classifiers and their recall values. R2UNet classifier has $77.9 \%$. This is one and only classifier has below $80 \%$ of recall value. Others are above $80 \%$ of recall value. SegNet and GoogleNet classifiers have $81.4 \%$ and $89.4 \%$. Rest of the classifiers have above $90 \%$. Our proposed model has highest recall value which is $97.15 \%$

The below diagram represents that the F1 score values of various classifiers. i.e., VGG-16 technique gives $75.3 \%$ of F1 Score value. Alex Net technique gives $81.3 \%$ of F1 Score. GoogleNet technique gives $89.1 \%$ of F1 Score value. CNN classifier gives the F1 Score value is $88.8 \%$, SegNet algorithm gives $84.6 \%$ of F1 Score value. R2UNet classifier gives the F1 Score value is $81.7 \%$ and Proposed_DeepIncepRC gives $97.25 \%$ of F1 Score value. 


\section{VARIOUS TECHNIQUES VS F1-SCORE}

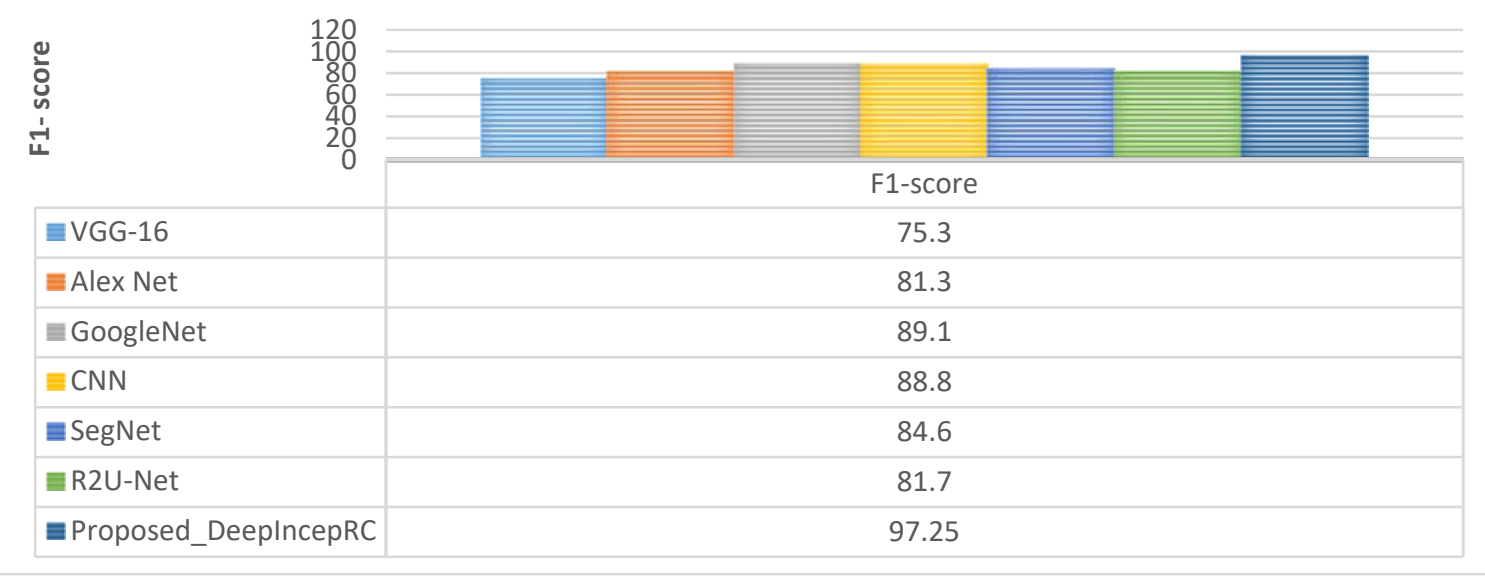

Figure 4: Various classifiers Vs F1- Score

The below diagram represents that the F1 score values of various classifiers. i.e., VGG-16 technique gives $75.3 \%$ of F1 Score value. Alex Net technique gives $81.3 \%$ of F1 Score. GoogleNet technique gives $89.1 \%$ of F1 Score value. CNN classifier gives the F1 Score value is $88.8 \%$, SegNet algorithm gives $84.6 \%$ of F1 Score value. R2UNet classifier gives the F1 Score value is $81.7 \%$ and Proposed_DeepIncepRC gives $97.25 \%$ of F1 Score value.

\section{Conclusion}

In this research clearly focuses the optimal solutions of accuracy, precision, recall and F1 score value provided by proposed system Proposed_DOOPIncepRC. Which is producing the $97.3 \%$ of accuracy level, $97.37 \%$ of precision level, $97.15 \%$ of recall value and $97.25 \%$ of F1 score value. These results are comparatively high with other models.

\section{REFERENCES}

[1] Bhattacherjee, Aindrila, "Classification approach for breast cancer detection using back propagation neural network: a study", Biomedical image analysis and mining techniques for improved health outcomes,pp.210-221, 2016.

[2] Trister, Andrew D, Diana SM Buist and Christoph I. Lee,"Will machine learning tip the balance in breast cancer screening?", JAMA oncology, pp.1463-1464, 2017.

[3] Prasath Alias Surendhar S, “A Review on Detecting the Breast Cancers using Various Algorithms”, pp.19362 - $19365,2020$.

[4] Hsieh, Sheau-Ling, "Design ensemble machine learning model for breast cancer diagnosis", Journal of medical systems,pp.2841-2847, 2012.

[5] Prasath Alias Surendhar S, "Early Diagnosis of Breast Cancer using Various Techniques", International Journal of Advanced Science and Technology,vol.29, no.4, pp. 563-572, 2020.

[6] Deniz, Erkan, "Transfer learning based histopathologic image classification for breast cancer detection", Health information science and systems, 2018.

[7] Osmanović, Ahmed, "Machine learning techniques for classification of breast cancer", World Congress on Medical Physics and Biomedical Engineering, 2019.

[8] N. Dhungel, G. Carneiro and A. P. Bradley, "Automated Mass Detection in Mammograms using Cascaded Deep Learning and Random Forests",International Conference on Digital Image Computing: Techniques and Applications (DICTA),pp.1-8, 2015.

[9] Y. Li, H. Chen, L. Zhang and L. Cheng, "Mammographic mass detection based on convolution neural network",24th International Conference on Pattern Recognition (ICPR), pp.3850-3855, 2018.

[10] N. Dhungel, G. Carneiro and A. P. Bradley, "A deep learning approach for the analysis of masses in mammograms with minimal user intervention", Medical Image Analysis, vol.37, pp.114-128, 2017.

[11] H. S. Oliveira, J. F. Teixeira and H. P. Oliveira, "Lightweight Deep Learning Pipeline for Detection, Segmentation and Classification of Breast Cancer Anomalies",International Conference on Image Analysis and Processing, pp. 707-715, 2019.

[12] Yan-yan SONG, Ying LU, Decision tree methods: applications for classification and prediction, Shanghai Archives of Psychiatry, 2015 Apr 25; 27(2): 130-135.

[13] NusaibahKh. Al-Salihy, Turgay IBRIKCI Classifying Breast Cancer by Using Decision Tree Algorithms, Proceedings of the $6^{\text {th }}$ International Conference on Software and Computer Applications, ACM , pp.144-148, 2017.

[14] PuneetYadav, RajatVarshney, Vishan Kumar Gupta, "Diagnosis of Breast Cancer using Decision Tree Models and SVM",International Research Journal of Engineering and Technology (IRJET), vol.5, no.3, 2018.

[15] Dr.NeerajBhargava, Girja Sharma Dr.RituBhargava, Manish Mathuria, "Decision Tree Analysis on J48 Algorithm for Data Mining", International Journal of Advanced Research in Computer Science and Software Engineering, vol.3, no.6, 2013.

[16] GaganjotKaur, AmitChhabra, "Improved J48 Classification Algorithm for the Prediction of Diabetes", International Journal of Computer Applications, vol.98, no.22, 2014.

[17] Ahmed IqbalPritom, AhadurRahmanMunshi, ShahedAnzarusSabab, ShihabuzzamanShihab, "Predicting Breast Cancer Recurrence using effective Classification and Feature Selection technique", $19^{\text {th }}$ International Conference on Computer and Information Technology, pp.18-20, 2016

[18] Dr.Nalini C, D.Meera, "Breast cancer prediction system using Data mining methods", International Journal of Pure and Applied Mathematics,vol.119, no.12, pp.10901-10911, 2018.

[19] RawaaAbdulridhaKadhim, "Classification and Predication of Breast Cancer Risk Factors Using Id3", International Journal Of Engineering And Science (IJES), vol.5,no.11, pp.29-33, 2016. 
[20] Sung-HyukCha , Charles Tappert, "A Genetic Algorithm for Constructing Compact Binary Decision Trees", Journal Of Pattern Recognition Research 1,pp.1-13, 2009.

[21] EminaAlickovic, AbdulhamitSubasi, "Breast cancer diagnosis using GA feature selection and Rotation Forest", Neural Computing and Applications, 2015

[22] Li Min Wang, Xiao Lin Li, Chun Hong Cao, Sen Yuan Miao, "Combing decision trees and naive bayes for classification", Elsevier, Knowledge Based Systems, pp.511-515, 2006.

[23] Yoav Freund, Lew mason, "The alternating decision tree algorithm", Proceeding of the Sixteenth International Conference on Machine Learning, pp.124-133, 1999.

[24] E. Venkatesan, T. Velmurugan, "Performance Analysis of Decision Tree Algorithms for Breast Cancer Classification", Indian Journal of Science and Technology, vol.8, no.29, 2015.

[25] MohamadBadr Al Snousy, Hesham Mohamed El- Deeb, KhaledBadran, Ibrahim Ali al Khlil, "Suite of decision-tree based classification algorithms on cancer gene expression data", Egyptian Informatics Journal,pp.73-82, 2011.

[26] Prasath Alias Surendhar S, "Cerebrum Tumour Classification Using Edge Detection And Cnn", International Journal of Advanced Science and Technology,vol.29, no.4s, pp. 553-562, 2020

[27] Ayyappan.G et al. A Novel Classification Approach -1 on Breast Tissue dataset, Indian Journal of Computer Science and Engineering (IJCSE), Volume No.9 Issue No.4 Aug-Sep 2018, Page No: 115-118, e-ISSN: 0976-5166, p-ISSN: 2231-3850.

[28] Ayyappan.G et al. Heart Disease Data Set Classifications: Comparisons of Correlation Co Efficient by Applying Various Functions, Indian Journal of Computer Science and Engineering (IJCSE), Volume No.9 Issue No.5 Oct-Nov 2018, Page No: 135-140, e-ISSN: 0976-5166, p-ISSN: 2231-3850.

[29] https://core.ac.uk/download/pdf/47139244.pdf

[30] http://peipa.essex.ac.uk/info/mias.html

[31] http://medicalresearch.inescporto.pt/breastresearch/index.php/Get_INbreast_Database 